\title{
EL ESTRÉS CALÓRICO AFECTA EL COMPORTAMIENTO REPRODUCTIVO Y EL DESARROLLO EMBRIONARIO TEMPRANO EN BOVINOS
}

\author{
Roger Molina-Coto ${ }^{1}$
}

\section{RESUMEN}

La ganadería bovina no se encuentra exenta de sufrir las consecuencias ambientales y en específico del fenómeno de cambio climático. Existe evidencia que en los últimos años las temperaturas están aumentando a nivel global. El bovino es un animal que tiene la capacidad de regular su temperatura corporal, sin embargo, cuando se encuentra en un ambiente fuera de su zona de confort comienza a experimentar retos metabólicos que generan consecuencias negativas a nivel productivo y reproductivo. En el caso de la reproducción de la hembra bovina, es claro que existen múltiples factores que se pueden ver afectados por estrés calórico, las cuales, en conjunto, conllevan a disminución de la fertilidad. Bajo condiciones de estrés calórico, la intensidad y duración del estro disminuye, a nivel ovárico se han encontrado consecuencias en la dinámica de ondas foliculares y alteración en la concentración de hormonas como estrógenos y progesterona. Esto afecta la competencia del ovocito y por tanto la tasa de concepción. A nivel embrionario los primeros estadios son vulnerables al estrés calórico y conforme se va desarrollando el embrión va adquiriendo tolerancia a dichas condiciones, sin embargo, la señal de reconocimiento materno fetal por parte del trofoblasto embrionario es débil. En general, el estrés calórico afecta la calidad del ovocito y produce pérdidas embrionarias tempranas que disminuyen la fertilidad de la vaca. Esta revisión describe las interrelaciones de los principales factores involucrados en la reproducción de la hembra bovina que son afectados por el estrés calórico.

Palabras clave: Estrés calórico, vacas, pérdidas embrionarias, dinámica folicular, comportamiento reproductivo

\footnotetext{
${ }^{1}$ Universidad de Costa Rica. Escuela de Zootecnia. San José, Costa Rica. Autor para correspondencia: roger.molina@ucr.ac.cr 


\section{ABSTRACT}

Heat stress affects reproductive behavior and early embryonic development in cattle. Bovine animals are not exempt from suffering the environmental challenges and specifically the consequences of climate change. There is evidence that in recent years temperatures are increasing globally. The bovine is an animal that is able to regulate its own body temperature, however, when it is facing an environment outside of its comfort zone, begins to experience metabolic challenges that generate negative consequences at productive and reproductive levels. In the case of female bovine reproduction, there are multiple factors that could be affected by heat stress, which, together, lead to a decrement in fertility. Under heat stress conditions, the intensity and duration of estrous decrease, at ovarian level, follicular dynamic is altered. Hormones such as estrogens and progesterone are affected as well. This affects the oocyte competence, therefore, the conception rate. At embryonic level, the first stages of development are vulnerable to heat stress but as the embryo develops, it acquires tolerance to these conditions, however, the signal of fetal maternal recognition that is given by the embryonic trophoblast is weak. Overall, heat stress affects the quality of the oocyte and produces early embryonic losses that decrease the fertility of the cow. This review describes the interrelationships of the main factors involved in the reproduction of the bovine female that are affected by the heat stress.

\section{Key words:}

Heat stress, cows, embryonic loss, follicular dynamic, reproductive behavior 


\section{INTRODUCCIÓN}

La zona climática intertropical en la cual se ubica Costa Rica, al igual que el resto de Centroamérica, se caracteriza por clima caliente y con regiones de clima vulnerable para la producción agropecuaria (Bouroncle et al., 2015). El cambio climático es uno de los principales retos a los que la humanidad se enfrenta durante los últimos años, por ejemplo, el clima comienza a ser extremo en lugares donde antes no lo era (Easterling et al., 2016). Factores asociados al cambio climático como el efecto invernadero, ozono, aerosoles y uso de la tierra, así como otros factores donde el ser humano tiene menor control, como variaciones solares y volcanes, agudizan el aumento de temperatura y precipitación (Easterling et al., 2016). En los últimos 66 años, a nivel global, se da una tendencia por días y noches más calientes, así como aumento de precipitación en algunas regiones o, por el contrario, menor precipitación. Esto genera riesgo para la producción agrícola y animal, por tanto, la seguridad alimentaria regional y mundial (Heim Jr, 2015).

Según datos del Censo Agropecuario del 2014, en Costa Rica se destinan 1.044.909,7 hectáreas para pastos, distribuidas en 37.171 fincas, área equivalente al $20 \%$ del territorio nacional. En estas fincas se mantiene una población de 538.593 bovinos para carne, 327.130 para leche y 409.889 animales de doble propósito. En el caso de los sistemas de producción de doble propósito y de carne, estos se ubican en su mayoría en zonas cálidas del país.

En el caso de los mamíferos, éstos animales tienen la capacidad de termoregularse, lo cual les permite mantener una temperatura corporal constante. En el caso específico de la vaca lechera, dicha temperatura es alrededor de $38,8{ }^{\circ} \mathrm{C}$ (West, 2003). El mismo autor reporta que la zona de confort para la vaca lechera va desde 5 a 20 grados Celsius, el animal en ésta zona puede regular su temperatura sin necesidad de invertir energía adicional. Para vacas lecheras, valores de 68 a 71 de índice de temperatura y humedad (ITH) comienzan a provocar efectos negativos por estrés calórico. El ITH es un índice que como su nombre lo indica, combina la temperatura y la humedad a la cual están expuestos los animales. La escala de ITH define puntos críticos o niveles de severidad del estrés calórico, en el rango de 72 a 79 se considera un estrés moderado, de 80 a 89 es un estrés de moderado a severo y de 90 a 98 es un estrés severo o incluso letal (Zimbelman y Collier, 2011). 
Collier et al. (1982) mostraron que la etapa fisiológica del bovino lechero afecta la definición de los puntos críticos dentro de un rango de temperatura sobre o bajo el cual el desempeño y la salud del animal se ven comprometidos. El animal adulto alcanza mayor tolerancia al frío, pero sucede lo contrario con la habilidad para tolerar el calor. La producción de leche en regiones tropicales, así como la alteración de factores ambientales, en conjunto con las características propias de la explotación lechera, hacen que los animales sufran las consecuencias del estrés provocado por el calor.

Lo anterior ha hecho que los productores del sector agropecuario se enfoquen en utilizar razas y variedades agrícolas adaptadas a dichas condiciones, sin embargo, en ocasiones parece no ser suficiente(Shannon y Motha, 2015). Ésta revisión tiene como objetivo caracterizar el efecto del estrés calórico sobre el comportamiento reproductivo, capacidad de establecimiento y mantenimiento de la preñez para sensibilizar sobre la necesidad de mitigación del estrés calórico en los bovinos.

\section{ASPECTOS GENERALES DE LA REPRODUCCION Y DESARROLLO EMBRIONARIO TEMPRANO}

El ciclo estral en el bovino comienza al iniciar la pubertad, etapa fisiológica del animal que se define por el inicio de la actividad ovárica que le brinda al animal la habilidad para preñarse (Diskiny Kenny, 2014). La pubertad se alcanza entre 10 y 12 meses para hembras bovinas de leche, entre 11 y 15 meses para ganado de engorde Bos taurus y entre 18 y 24 meses para las razas cebuinas (Hafez, 1996). El ciclo estral se refiere a la presencia del estro de manera periódica, normalmente entre 18 a 24 días, sin embargo, en vacas de leche, su longitud es tema de discusión (Remnant et al., 2016). El estro en la vaca se caracteriza por un incremento de movimiento, vocalización, elevación de la cola, intentos de monta y quietud a la monta (Senger, 2003). La mayor actividad o expresión de celo se da durante la noche o las horas más frescas del día (Bolocan, 2009). Para que el ciclo estral se presente, es necesario que el ciclo ovárico esté activo. El ciclo ovárico se refiere a la fisiología y anatomía relacionada con el desarrollo y función de estructuras ováricas donde se incluyen las etapas de proestro, estro, metaestro y diestro. El mismo es dirigido por hormonas provenientes del eje hipotálamo-hipófisis que actúan sobre las gónadas femeninas y de hormonas producidas como respuesta a las mismas (Senger, 2003). La duración del estro va desde 9 a 28 horas, sin embargo, la forma de medirlo puede afectar la 
duración. Una medida más homogénea es el tiempo de receptividad a la monta que es de alrededor de 9,5 horas (Sepúlveda y Rodero, 2003).

La ovulación en la vaca se clasifica como espontánea y se presenta entre 24 a 32 horas posterior al inicio del estro (Senger, 2003). Si se da un servicio, sea natural o artificial, y si el servicio coincide con la ovulación, se puede dar la fertilización. Una vez que se haya dado la fertilización, comienza el desarrollo embrionario. La fertilización ocurre en el oviducto, evento que permite la formación del zigoto. Posterior a ésta etapa, el embrión es transportado desde el oviducto hacia el útero y simultáneamente se divide. El embrión entra al útero alrededor del día 4 post fertilización con un estadio de 16 células. Posteriormente, se da el proceso de compactación celular y la unión entre células en el estadio de mórula. Alrededor del día 7, la mórula se convierte en blastocito en donde se diferencia una masa celular central y trofoblasto, éste último es el encargado de formar la placenta y la masa celular central es la parte del embrión que da lugar al feto (Lonergan et al., 2016).

El embrión comienza a dividirse dentro de la zona pelúcida del ovocito que le dio origen. Cuando el embrión está en estado de blastocito sale de esa zona pelúcida lo cual sucede alrededor del día 8. Además, el embrión sufre un proceso de elongación, pasando desde su forma esferoidal al día 7 a una forma ovoide alrededor del día 12, luego alcanza una forma elongada entre los días 16 y 17 para seguir con el subsecuente desarrollo embrionario, organogénesis y desarrollo fetal (Degrelle et al., 2005).

\section{COMPORTAMIENTO REPRODUCTIVO ANTE PERIODOS DE ESTRÉS CALORICO}

\section{Respuesta en la preñez y el comportamiento reproductivo}

En la industria lechera, la producción de terneras como futuros reemplazos y la producción de leche per se son metas fundamentales (McParland et al., 2008). Para esto la hembra bovina debe estar en condiciones adecuadas que le permitan desarrollar ciclos estrales y ováricos regulares para generar preñeces tanto por medios naturales como artificiales. La vaca durante su lactancia y en condiciones de estrés calórico se enfrenta a un doble desafío. West (2003), afirma que las vacas lactantes generan, producto de su metabolismo, de 27 a $48 \%$ más calor que vacas no lactantes, esto debido a que las vacas productoras de leche tienen altos requerimientos de 
nutrientes que las obliga a consumir más alimento y por tanto se genera mayor calor metabólico. Sartori et al., (2002) determinaron que las vacas lactantes se ven más comprometidas ante el estrés calórico que las novillas, lo que confirma que la tasa metabólica que produce la lactancia afecta la respuesta de los animales ante el estrés calórico.

En regiones tropicales, subtropicales y sitios de marcada estacionalidad, se demostró la relación negativa entre la temperatura ambiental y la tasa de preñez en vacas de leche. En Florida, USA, (Hansen yArechiga (1999) determinaron una disminución mayor a $15 \%$ en el porcentaje de preñez entre los meses calientes y los meses fríos. Estos datos coinciden con López-Gatius et al., (2006)y con los datos de Poock et al., (2015), donde se determinó una disminución en la tasa de preñez de vacas inseminadas a tiempo fijo de $45 \%$, en la temporada fría, hasta $30 \%$ en la temporada caliente.

En sistemas de producción donde la detección de celos es la base para definir el momento de servicio para las hembras bovinas, las condiciones de estrés calórico en el animal son capaces de reducir cerca de $20 \%$ la cantidad de celos detectados (Hansen, 2005). Además, la intensidad con la cual se dan las manifestaciones de celo disminuye conforme aumenta el estrés por calor. Bolocan (2009) demostró que la claridad de los síntomas de estro disminuye de $83,3 \%$ a $16,7 \%$ cuando se pasa de 64,4 a 78,9 en el ITH, además, definió que la actividad de monta en novillas Holstein se concentra en las horas más frescas del día que coinciden con las horas de descanso de los vaqueros. Con el estrés calórico, no sólo disminuye la intensidad sino también la duración del estro, aumentan las ovulaciones silenciosas y la incidencia de anestro (Wakayo et al., 2015). La intensidad del estro, medida como actividad (pasos) fue 2,34 veces menor en verano que cuando se midió durante el invierno (Sakatani et al., 2012b). La tasa de no retorno a celo (NRR) durante 90 días para el primer servicio, es decir, vacas que quedaron preñadas después del servicio, varió entre 15,8 y 23,6\% entre animales expuestos a estrés calórico y animales en ambiente termoneutral, donde los animales expuestos al estrés calórico tuvieron menores NRR que aquellos animales servidos en ambiente termoneutral (Al-Katanani et al., 1999).

\section{Respuesta de la fisiología reproductiva}

Las estructuras ováricas, así como concentraciones hormonales locales o sistémicas se evalúan para conocer la forma en la cual el estrés calórico afecta la función reproductiva. Por ejemplo, Wilson et al., (1998b) bajo condiciones experimentales 
controladas $\left(60 \%\right.$ de humedad relativa, $19^{\circ} \mathrm{C}$ (control) versus $\left.29^{\circ} \mathrm{C}\right)$ no encontraron diferencias en el diámetro del folículo dominante en vacas Holstein. Sin embargo, Wilson et al. (1998a) encontraron que el diámetro del folículo dominante al final ciclo estral, en novillas bajo estrés calórico $\left(60 \%\right.$ humedad relativa, $21^{\circ} \mathrm{C}$ (control) versus $29^{\circ} \mathrm{C}$ ), fue significativamente menor que en el grupo termoneutral. A pesar de la diferente respuesta en el tamaño del folículo, Wilson et al., (1998b) y Wilson et al., (1998a) encontraron que la concentración de estradiol en plasma fue significativamente menor tanto para vacas como novillas en condiciones de estrés calórico. Por otro lado, en dos experimentos sobre estrés calórico (Roth et al., 2000; de Castro e Paula et al., 2008), donde las evaluaciones se hacen a nivel de ambiente natural o usando mitigadores de estrés calórico (ventilación, sombras, aspersores),no se encontraron diferencias en la concentración de estradiol en vacas con o sin estrés calórico.

La dinámica folicular es afectada por el estrés calórico, el efecto de dominancia decrece, hay una emergencia temprana del folículo preovulatorio y carencia en la regresión de los folículos subordinados (Wolfenson et al., 1995).El efecto del estrés calórico sobre la dominancia en el desarrollo de ondas foliculares fue también confirmado por Trout et al., (1998). En un experimento in vitro, donde se cultivaron fragmentos ováricos durante 7 días, el complejo cúmulus-ovocito (COC) y ovocitos aislados fueron sometidos a estrés calórico durante las primeras 12 horas del experimento. Se encontró que el tratamiento térmico incrementó la activación de folículos primordiales, redujo la maduración nuclear del ovocito en los COC, alteró la secreción de hormonas esteroideas y aumentó la producción de radicales oxidativos (Paes et al., 2016). Se estima que los efectos del estrés calórico sobre la dinámica ovárica tienen consecuencias en el corto y largo plazo y que para que la dinámica ovárica se normalice deben pasar de 2 a 3 ciclos estrales desde la última afectación por calor (Roth, 2015).

Wilson et al. (1998b) encontraron que la vida del cuerpo lúteo en vacas cíclicas en condiciones de estrés calórico es más larga y que las concentraciones de progesterona se mantienen significativamente más altas del día 17 al 21 del ciclo estral. El comportamiento del cuerpo lúteo de vida larga observado en vacas bajo estrés calórico, fue el mismo obtenido cuando la evaluación se realizó con novillas, la única diferencia es que las concentraciones altas de progesterona fueron de los días 16 al 20 del ciclo estral. El efecto para vacas y novillas es congruente si se toma en cuenta que la longitud del ciclo estral en novillas tiende a ser más corta. El efecto sobre las concentraciones de progesterona fue informado en periodos similares por 
Trout et al., (1998) pero en condiciones de campo. Casos contrarios a éste comportamiento, cuatro investigaciones describen la no alteración de las concentraciones de progesterona bajo condiciones de estrés calórico (de Castro e Paula et al., 2008;Gilad et al., 1993; Roth et al., 2000).

En cuanto a concentraciones de hormonas gonatrópicas, Roth et al., (2000) encontraron una menor concentración de FSH en vacas bajo estrés calórico que en el grupo control, sin embargo, Gilad et al., (1993) no detectaron diferencias en las concentraciones de FSH y LH en vacas con o sin estrés calórico.

El ambiente uterino también se ve comprometido bajo condiciones de estrés calórico. La secreción histotrófica es fundamental para el desarrollo embrionario temprano. En condiciones in vitro, se determinó que el estrés calórico altera el perfil proteico del histotrofo y además presentó mayores concentraciones de prostaglandinas $E$ y $F_{2 \alpha}$ (Putney et al., 1988). La alteración del perfil proteico en el útero en condiciones de estrés calórico fue confirmado por evaluación de su transcriptoma, la expresión de 637 genes se alteró cuando se comparó tejido uterino de animales durante el verano o el otoño con una importante expresión de proteínas de choque térmico de las muestras tomadas durante el verano (Sakumoto et al., 2015).

\section{Respuesta del desarrollo embrionario temprano}

Los efectos del estrés calórico también se evalúan durante el desarrollo embrionario temprano, para ello, es común el uso de modelos de producción de embriones in vivo e in vitro. Por ejemplo, Hansen (2005) postula que los ovocitos en el folículo, la fecundación y los primeros dos días de desarrollo embrionario son sensibles al estrés calórico; al tercer y cuarto día el embrión adquiere cierta tolerancia, misma que aumenta al quinto día. Esto es reforzado por Edwards y Hansen (1997) quienes compararon el desarrollo embrionario en condiciones térmicas de 39 contra $41^{\circ} \mathrm{C}$ durante las etapas específicas de: dos células, 4-8 células y mórula compacta. No obtuvieron desarrollo de blastocitos al día 7 de los embriones estresados en etapa de dos células, obtuvieron un menor desarrollo de blastocitos al día 7 de los embriones provenientes del grupo tratado en la etapa de 4 a 8 células y la misma cantidad de blastocitos provenientes de los grupos tratados en el estadio de mórula. Sakatani et al. (2012a) encontraron resultados similares con la aplicación de un tratamiento de $40^{\circ} \mathrm{C}$ durante 24 horas en un cultivo de mórulas, el cual no tuvo ningún efecto adverso en el desarrollo de blastocitos mientras que sí hubo una disminución significativa en el 
porcentaje de blastocitos desarrollados cuando el mismo tratamiento se aplicó a nivel de zigotos.

En estadios embrionarios más desarrollados (embrión elongado), se encontró que embriones bajo estrés calórico disminuyeron la secreción de la proteína trofoblastica 1 conocida también como interferón tau, la cual es la señal del reconocimiento maternofetal (Putney et al., 1988).

El componente genético afecta la tolerancia al estrés calórico desde el desarrollo embrionario temprano. Paula-Lopes et al., (2003) indujo estrés calórico en embriones de 8 células de las razas Senepol, Holstein, Angus y Brahman y encontró que los embriones de Holstein y Angus presentaron significativamente menor desarrollo en esas condiciones que los embriones de Senepol o Brahman. Hernández-Cerón et al., (2004) coinciden con Paula-Lopes et al., (2003) puesto que utilizaron embriones de 8 a 16 células de las razas Angus, Brahman y Romosinuano y encontraron que los embriones de la raza Angus desarrollaron menos que los de las razas Brahman o Romosinuano.

En la Figura 1 se presenta un diagrama que resume las principales vías por las cuales el estrés calórico afecta la fertilidad de la vaca. se muestra la interrelación de factores que influyen sobre ejes principales que finalmente, de manera directa o indirecta, convergen para disminuir la fertilidad de la hembra bovina bajo condiciones de estrés calórico. 


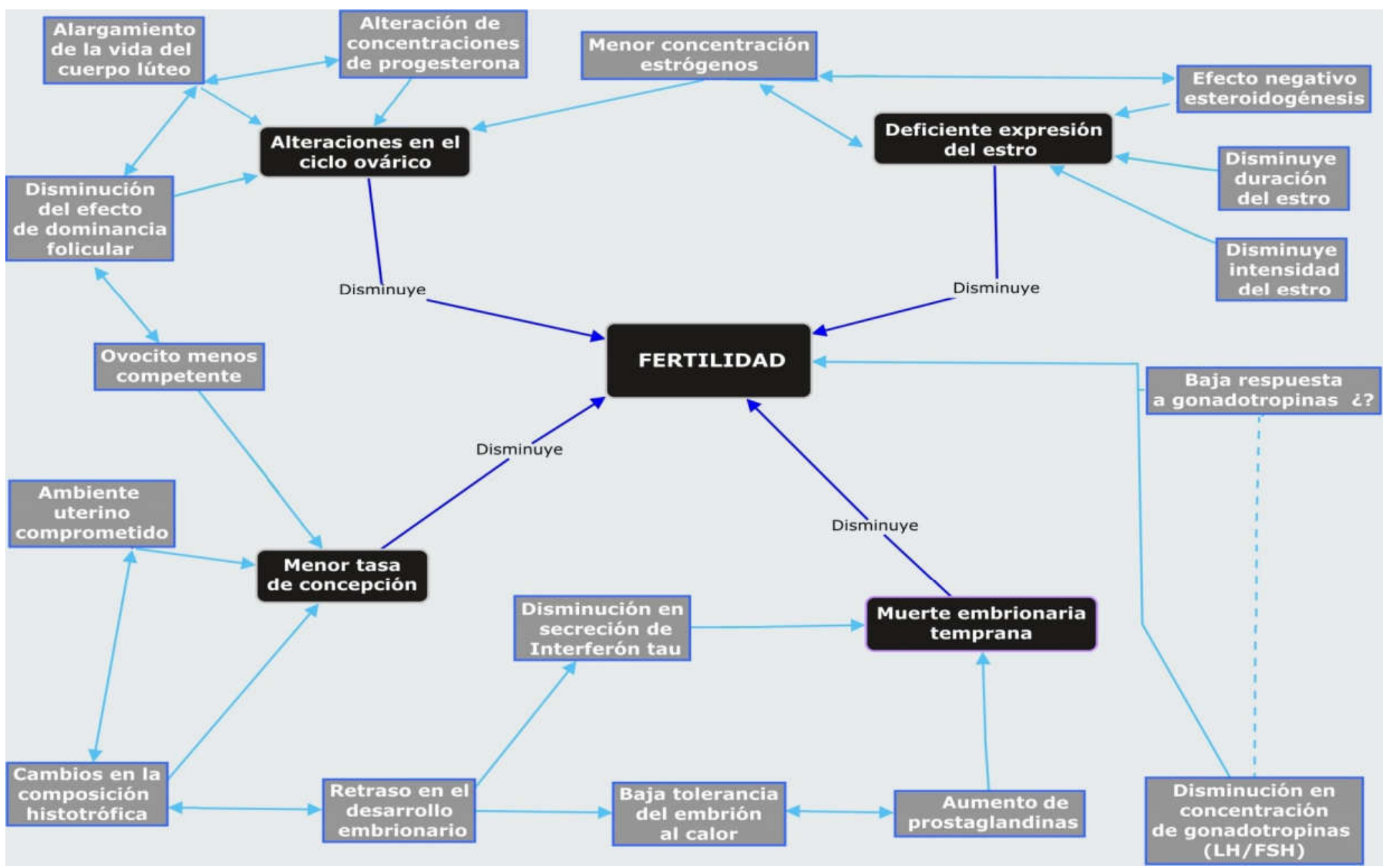

Figura 1. Relación de los principales factores por los cuales el estrés calórico afecta el comportamiento reproductivo, el desarrollo embrionario temprano y la fertilidad en la hembra bovina. 
Existe además un factor de consumo de alimento de los animales que no fue contemplado en esta revisión, pero que también afecta de manera directa e indirecta la reproducción puesto que animales bajo estrés calórico disminuyen el consumo de alimento y por tanto de nutrientes vitales para las funciones de mantenimiento, crecimiento, producción y reproducción (Zimbelman yCollier, 2011).

\section{ALTERNATIVAS PARA MITIGAREL ESTRÉS CALORICO SOBRE LA REPRODUCCION}

El problema causado por el estrés calórico para la ganadería es evidente, para mitigar los efectos del estrés calórico existen diversas alternativas como el uso de sombras naturales o artificiales (Schütz et al., 2014), mecanismos de ventilación y aspersores (Ortiz et al., 2015), así como el uso de razas o cruces mejor adaptados a condiciones calóricas(Alfonzo et al., 2016), incluso haciendo uso de herramientas moleculares como la selección genómica (Garner et al., 2016). De igual forma existen aspectos de manejo que pueden mitigar los efectos del estrés calórico como por ejemplo, el uso de métodos de detección de celo eficientes, uso de protocolos de sincronización de celo para inseminación a tiempo fijo (Hansen et al., 2001), producción y transferencia de embriones (Rutledge, 2001) y manejo del ganado durante horas y épocas frescas. Es necesario mencionar que existen estrategias nutricionales que también mitigan el estrés calórico en los bovinos (West, 1999).

\section{CONSIDERACIONES FINALES}

El estrés calórico causa disminución en la fertilidad de la hembra bovina. Es un fenómeno en crecimiento de la mano con las tendencias de cambio climático reportadas. La afectación de la reproducción en la vaca debido al estrés calórico es multifactorial, aunque en algunos casos, la literatura aun no deja del todo claro la forma en la cual el calor afecta, es claro que el estrés calórico causa alteraciones en la dinámica folicular, perfiles hormonales, ambiente uterino y desarrollo embrionario temprano que finalmente disminuyen la fertilidad de la vaca.

Existen métodos que el ganadero puede utilizar para mitigar los efectos del estrés calórico y además, brindarle a sus animales ambientes confortables que le ayuden en su termorregulación y productividad; además, el estrés por calor es un componente a tomar en cuenta tanto para la investigación en busca de la mitigación de sus efectos como para el manejo diario de las explotaciones ganaderas. 


\section{LITERATURA CITADA}

Alfonzo, E.P.M., M.V.G.B. da Silva. D. dos S. Daltro, M.T. Stumpf, V.C. Dalcin, G. Kolling, V. Fischer, y C.M. McManus. 2016. Relationship between physical attributes and heat stress in dairy cattle from different genetic groups. Int. J. Biometeorol. 60(2): 245-253.

Al-Katanani, Y.M., D.W. Webb, y P.J. Hansen. 1999. Factors affecting seasonal variation in 90-day nonreturn rate to first service in lactating Holstein cows in a hot climate. J. Dairy Sci. 82(12): 2611-2616.

Bolocan, E. 2009. EFFECTS OF HEAT STRESS ON SEXUAL BEHAVIOR IN HEIFERS STUDIUL COMPORTAMENTULUI SEXUAL LA JUNINCI ÎN CONDITII DE STRES TERMIC. Disponible en http://www.academia.edu/download/6755785/bolocan.pdf (consultado el 21 de Septiembre. 2016).

Bouroncle, C., P. Imbach, P. Läderach, B. Rodríguez, C. Medellín, E. Fung, M.R. Martínez-Rodríguez, y C.I. Donatti. 2015. La agricultura de Costa Rica y el cambio climático: ¿Dónde están las prioridades para la adaptación? Disponible enhttps://cgspace.cgiar.org/handle/10568/45942 (Consultado el 30 de Noviembre. 2016).

de Castro e Paula, L.A., J. Andrzejewski, D. Julian, L.J. Spicer, y P.J. Hansen. 2008. Oxygen and steroid concentrations in preovulatory follicles of lactating dairy cows exposed to acute heat stress. Theriogenology 69(7): 805-813.

Collier, R.J., D.K. Beede, W.W. Thatcher, L.A. Israel, y C.J. Wilcox. 1982. Influences of Environment and Its Modification on Dairy Animal Health and Production1. J. Dairy Sci. 65(11): 2213-2227.

Degrelle, S.A., E. Campion, C. Cabau, F. Piumi, P. Reinaud, C. Richard, J.-P. Renard, y I. Hue. 2005. Molecular evidence for a critical period in mural trophoblast development in bovine blastocysts. Dev. Biol. 288(2): 448-460.

Diskin, M.G., y D.A. Kenny. 2014. Optimising reproductive performance of beef cows and replacement heifers. animal 8(s1): 27-39.

Easterling, D.R., K.E. Kunkel, M.F. Wehner, y L. Sun. 2016. Detection and attribution of climate extremes in the observed record. Weather Clim. Extrem. 11: 17-27.

Garner, J.B., M.L. Douglas, S.R.O. Williams, W.J. Wales, L.C. Marett, T.T.T. Nguyen, C.M. Reich, y B.J. Hayes. 2016. Genomic Selection Improves Heat Tolerance in Dairy Cattle. Sci. Rep. 6 Disponible en http://www.ncbi.nlm.nih.gov/pmc/articles/PMC5040955/ (Consultado el 30 de Noviembre. 2016).

Gilad, E., R. Meidan, A. Berman, Y. Graber, y D. Wolfenson. 1993. Effect of heat stress on tonic and $\mathrm{GnRH}$-induced gonadotrophin secretion in relation to concentration of oestradiol in plasma of cyclic cows. J. Reprod. Fertil. 99(2): 315-321. 
Hansen, P.J., y C.F. Arechiga. 1999. Strategies for managing reproduction in the heatstressed dairy cow. J. Anim. Sci. 77: 36.

Hansen, P.J., M. Drost, R.M. Rivera, F.F. Paula-Lopes, Y.M. Al-Katanani, C.E. Krininger, y C.C. Chase. 2001. Adverse impact of heat stress on embryo production: causes and strategies for mitigation. Theriogenology 55(1): 91-103.

Hansen, P.J. 2005. Managing the heat-stressed cow to improve reproduction. p. 9-11. In Proceedings of the 7th Western Dairy Management Conference. Marzo. 2005

Heim Jr., R.R. 2015. An overview of weather and climate extremes - Products and trends. Weather Clim. Extrem. 10, Part B: 1-9.

Hernández-Cerón, J., C.C. Chase Jr, y P.J. Hansen. 2004. Differences in Heat Tolerance Between Preimplantation Embryos from Brahman, Romosinuano, and Angus Breeds*. J. Dairy Sci. 87(1): 53-58.

Lonergan, P., T. Fair, N. Forde, y D. Rizos. 2016. Embryo development in dairy cattle. Theriogenology 86(1): 270-277.

López-Gatius, F., P. Santolaria, A. Martino, F. Delétang, y F. De Rensis. 2006. The effects of $\mathrm{GnRH}$ treatment at the time of $\mathrm{Al}$ and 12 days later on reproductive performance of high producing dairy cows during the warm season in northeastern Spain. Theriogenology 65(4): 820-830.

McParland, S., L. Shalloo, y D.P. Berry. 2008. Evaluation and development of animal breeding in Ireland. Teagasc.

Ortiz, X.A., J.F. Smith, F. Villar, L. Hall, J. Allen, A. Oddy, A. al-Haddad, P. Lyle, y R.J. Collier. 2015. A comparison of 2 evaporative cooling systems on a commercial dairy farm in Saudi Arabia. J. Dairy Sci. 98(12): 8710-8722.

Paes, V.M., L.A. Vieira, H.H.V. Correia, N.A.R. Sa, A.A.A. Moura, A.D. Sales, A.P.R. Rodrigues, D.M. Magalhães-Padilha, F.W. Santos, G.A. Apgar, C.C. Campello, L.S.A. Camargo, y J.R. Figueiredo. 2016. Effect of heat stress on the survival and development of in vitro cultured bovine preantral follicles and on in vitro maturation of cumulus-oocyte complex. Theriogenology 86(4): 994-1003.

Paula-Lopes, F.F., C. Cc, Y.M. Al-Katanani, K. Ce, R.M. Rivera, S. Tekin, A.C. Majewski, O.M. Ocon, T.A. Olson, y P.J. Hansen. 2003. Genetic divergence in cellular resistance to heat shock in cattle: differences between breeds developed in temperate versus hot climates in responses of preimplantation embryos, reproductive tract tissues and lymphocytes to increased culture temperatures. Reproduction 125(2): 285-294.

Poock, S.E., W.R. Lamberson, y M.C. Lucy. 2015. Effect of different gonadorelin $(\mathrm{GnRH})$ products used for the first or resynchronized timed artificial insemination on pregnancy rates in postpartum dairy cows. Theriogenology 84(4): 504-508.

Putney, D.J., J.R. Malayer, T.S. Gross, W.W. Thatcher, P.J. Hansen, y M. Drost. 1988. Heat stress-induced alterations in the synthesis and secretion of proteins and prostaglandins by cultured bovine conceptuses and uterine endometrium. Biol. Reprod. 39(3): 717-728. 
Remnant, J., M. Green, J. Huxley, y C. Hudson. 2016. What is the normal estrous cycle length for the modern dairy cow? Theriogenology 86(9): 2334.

Roth, Z., R. Meidan, R. Braw-Tal, y D. Wolfenson. 2000. Immediate and delayed effects of heat stress on follicular development and its association with plasma FSH and inhibin concentration in cows. J. Reprod. Fertil. 120(1): 83-90.

Roth, Z. 2015. Physiology and endocrinology symposium: Cellular and molecular mechanisms of heat stress related to bovine ovarian function. J. Anim. Sci. 93(5): 2034-2044.

Rutledge, J.J. 2001. Use of embryo transfer and IVF to bypass effects of heat stress. Theriogenology 55(1): 105-111.

Sakatani, M., N.V. Alvarez, M. Takahashi, y P.J. Hansen. 2012a. Consequences of physiological heat shock beginning at the zygote stage on embryonic development and expression of stress response genes in cattle. J. Dairy Sci. 95(6): 3080-3091.

Sakatani, M., A.Z. Balboula, K. Yamanaka, y M. Takahashi. 2012b. Effect of summer heat environment on body temperature, estrous cycles and blood antioxidant levels in Japanese Black cow. Anim. Sci. J. 83(5): 394-402.

Sakumoto, R., K.-G. Hayashi, S. Saito, H. Kanahara, K. Kizaki, y K. Iga. 2015. Comparison of the global gene expression profiles in the bovine endometrium between summer and autumn. J. Reprod. Dev. 61(4): 297-303.

Sartori, R., R. Sartor-Bergfelt, S.A. Mertens, J.N. Guenther, J.J. Parrish, y M.C. Wiltbank. 2002. Fertilization and Early Embryonic Development in Heifers and Lactating Cows in Summer and Lactating and Dry Cows in Winter. J. Dairy Sci. 85(11): 2803-2812.

Schütz, K.E., N.R. Cox, y C.B. Tucker. 2014. A field study of the behavioral and physiological effects of varying amounts of shade for lactating cows at pasture. J. Dairy Sci. 97(6): 3599-3605.

Senger, P.L. 2003. Pathways to pregnancy and parturition. Second Revised Edition. Current Conceptions Inc., NE.

Sepúlveda, N., y E. Rodero. 2003. Comportamiento sexual durante el estro en vacas lecheras. Interciencia 28(9): 500-503.

Shannon, H.D., y R.P. Motha. 2015. Managing weather and climate risks to agriculture in North America, Central America and the Caribbean. Weather Clim. Extrem. 10, Part A: 50-56.

Trout, J.P., L.R. McDowell, y P.J. Hansen. 1998. Characteristics of the estrous cycle and antioxidant status of lactating Holstein cows exposed to heat stress. J. Dairy Sci. 81(5): 1244-1250.

Wakayo, B.U., P.S. Brar, y S. Prabhakar. 2015. Review on mechanisms of dairy summer infertility and implications for hormonal intervention. Open Vet. J. 5(1): 6-10. 
West, J.W. 1999. Nutritional strategies for managing the heat-stressed dairy cow. J. Anim. Sci. 77 Suppl 2: 21-35.

West, J.W. 2003. Effects of Heat-Stress on Production in Dairy Cattle. J. Dairy Sci. 86(6): 2131-2144.

Wilson, S.J., C.J. Kirby, A.T. Koenigsfeld, D.H. Keisler, y M.C. Lucy. 1998a. Effects of Controlled Heat Stress on Ovarian Function of Dairy Cattle. 2. Heifers1. J. Dairy Sci. 81(8): 2132-2138.

Wilson, S.J., R.S. Marion, J.N. Spain, D.E. Spiers, D.H. Keisler, y M.C. Lucy. 1998b. Effects of Controlled Heat Stress on Ovarian Function of Dairy Cattle. 1. Lactating Cows1. J. Dairy Sci. 81(8): 2124-2131.

Wolfenson, D., W.W. Thatcher, L. Badinga, J.D. Savio, R. Meidan, B.J. Lew, R. BrawTal, y A. Berman. 1995. Effect of heat stress on follicular development during the estrous cycle in lactating dairy cattle. Biol. Reprod. 52(5): 1106-1113.

Zimbelman, R.B., y R.J. Collier. 2011. Feeding Strategies for High-Producing Dairy Cows During Periods of Elevated Heat and Humidity. ResearchGate. Disponibleenhttps://www.researchgate.net/publication/268380843_Feeding_Str ategies_for_HighProducing_Dairy_Cows_During_Periods_of_Elevated_Heat_a nd_Humidity (Consultado el 21 de Septiembre. 2016). 